\title{
Emerging Plasmodium vivax resistance to chloroquine in South America: an overview
}

\author{
Lígia Antunes Gonçalves ${ }^{1,2}$, Pedro Cravo ${ }^{3}$, Marcelo Urbano Ferreira ${ }^{2 /+}$ \\ 'Latin America Initiative, Worldwide Antimalarial Resistance Network 2 Departamento de Parasitologia, \\ Instituto de Ciências Biomédicas, Universidade de São Paulo, São Paulo, SP, Brasil \\ ${ }^{3}$ Instituto de Patologia Tropical e Saúde Pública, Universidade Federal de Goiás, Goiânia, GO, Brasil
}

\begin{abstract}
The global emergence of Plasmodium vivax strains resistant to chloroquine (CQ) since the late 1980s is complicating the current international efforts for malaria control and elimination. Furthermore, CQ-resistant vivax malaria has already reached an alarming prevalence in Indonesia, East Timor and Papua New Guinea. More recently, in vivo studies have documented CQ-resistant P. vivax infections in Guyana, Peru and Brazil. Here, we summarise the available data on CQ resistance across P. vivax-endemic areas of Latin America by combining published in vivo and in vitro studies. We also review the current knowledge regarding the molecular mechanisms of CQ resistance in P. vivax and the prospects for developing and standardising reliable molecular markers of drug resistance. Finally, we discuss how the Worldwide Antimalarial Resistance Network, an international collaborative effort involving malaria experts from all continents, might contribute to the current regional efforts to map CQ-resistant vivax malaria in South America.
\end{abstract}

Key words: Plasmodium vivax - chloroquine - resistance - Latin America

Plasmodium vivax, the most widespread human malaria parasite, is estimated to cause up to 390 million clinical cases each year in Latin America, the Middle East, Central, South and Southeast Asia, Oceania and East Africa (Price et al. 2007, Battle et al. 2012), locations where 2.49 billion people are currently at risk of infection (Gething et al. 2012). Nearly half a million slide-confirmed malaria infections ( $80 \%$ due to $P$. vivax) are annually reported in 21 endemic countries across the Americas and the Caribbean (Arevalo-Herrera et al. 2012, da Silva-Nunes et al. 2012), with 137 million people at risk of malaria (Gething et al. 2012). Nonetheless, these figures are likely to be underestimates because of limitations in the coverage of malaria notification and diagnosis in many endemic areas. Nine Amazonian countries (Bolivia, Brazil, Colombia, Ecuador, Guyana, French Guyana, Peru, Suriname and Venezuela) together account for $90 \%$ of these infections.

The emergence of $P$. vivax strains resistant to chloroquine (CQ), which was first documented in Papua New Guinea (PNG) in 1989 (Rieckmann et al. 1989), complicates the current international efforts for malaria control and elimination. CQ resistance in vivo is formally defined as the persistence of asexual $P$. vivax blood stages despite adequate whole blood or plasma levels of CQ and its main active metabolite, desethylchloroquine (DCQ) (Baird et

doi: 10.1590/0074-0276130579

Financial support: Bill and Melinda Gates Foundation (to WWARN) MUF is supported by a senior research fellowship from CNPq.

+Corresponding author: muferrei@usp.br

Received 13 December 2013

Accepted 14 March 2014 al. 1997). By strictly applying this definition, $P$. vivax CQ resistance has been confirmed largely through the malaria endemic world, reaching an alarming prevalence in Indonesia and PNG (Baird 2009, Baird et al. 2012).

The first published reports of CQ-resistant vivax malaria in Latin America were from Colombia (Arias \& Corredor 1989) and Brazil (Garavelli \& Corti 1992); however, antimalarial treatment had not been supervised and drug levels were not measured in either of these early studies. Consequently, it was only in 1996 that CQ resistance was formally documented in a $P$. vivax strain from this region. Briefly, a Canadian patient returning from Guyana, who had been treated with CQ $(25 \mathrm{mg} / \mathrm{kg}$ over 3 days) and primaquine (PQ) (15 mg base/day for 14 days), had a parasite recrudescence on day 11 of $P Q$ treatment. His whole-blood CQ levels were in the therapeutic range $(>100 \mathrm{ng} / \mathrm{mL})$ at the time of treatment failure. A second patient, also returning from Guyana, had a late recurrence (6 weeks after initial CQ-PQ treatment), again with therapeutic blood levels of CQ at presentation (Phillips et al. 1996). Subsequent reports of $P$. vivax CQ resistance originated from Colombia [3 parasite recurrences among 27 patients (11.1\%)] (Soto et al. 2001) and Peru [4 recurrences among 212 patients (1.9\%)] (Ruebush et al. 2003) (Figure). The patients enrolled in these studies were treated with $25 \mathrm{mg}$ of $\mathrm{CQ} / \mathrm{kg}$ over three days, with no PQ being co-administered, but CQ resistance was confirmed in only two of them (both from Peru) by measuring drug levels at the time of recurrence.

More recently, two additional reports of in vivo CQ resistance in $P$. vivax were from Manaus, the major Brazilian port city in the Amazon Basin. In the first study, 11 of $109(10.1 \%)$ patients treated with CQ alone (25 $\mathrm{mg} / \mathrm{kg}$ over 3 days) had $P$. vivax recrudescence despite adequate plasma levels of CQ (de Santana Filho et al. 2007). Subsequently, another single-arm clinical trial 
in Manaus documented $P$. vivax recurrences in seven of $135(5.2 \%)$ patients treated with CQ $(25 \mathrm{mg} / \mathrm{kg}$ over 3 days) and PQ (30 mg base/day for 7 days); all of them had CQ levels above $100 \mathrm{ng} / \mathrm{mL}$ of whole blood at the time of recurrence (Marques et al. 2013). These studies indicate that $P$. vivax CQ resistance has occurred in at least three Latin American countries (Guyana, Peru and Brazil), affecting patients treated with either CQ alone or with a CQ-PQ combination.

However, other recent clinical trials have failed to confirm $P$. vivax CQ resistance in Guyana (Baird et al. 2002), Bolivia (Añez et al. 2012) and Colombia (Castillo et al. 2002, Alvarez et al. 2006, Carmona-Fonseca \& Maestre 2009). The patients treated with CQ alone who had treatment failures in Guayaramerín ( 8 of 79 subjects followed over 28 days) and Riberalta (5 of 81 subjects followed over 28 days), both in Bolivia, had blood levels of CQ and DCQ $<70 \mathrm{ng} / \mathrm{mL}$ at the time of parasite recurrence; as a consequence, $\mathrm{CQ}$ resistance could not be confirmed (Añez et al. 2012). Furthermore, one late treatment failure (on day 28 of CQ - only treatment) was recently observed in a small study (20 patients) in Tarapacá, Colombia, but the CQ concentration had not been measured at the time of parasite recrudescence (Osorio et al. 2007). Although CQ treatment failures have been reported in these study sites in Bolivia (Añez et al. 2012) and Colombia (Osorio et al. 2007) (Figure), the available data do not provide evidence for in vivo CQ resistance.

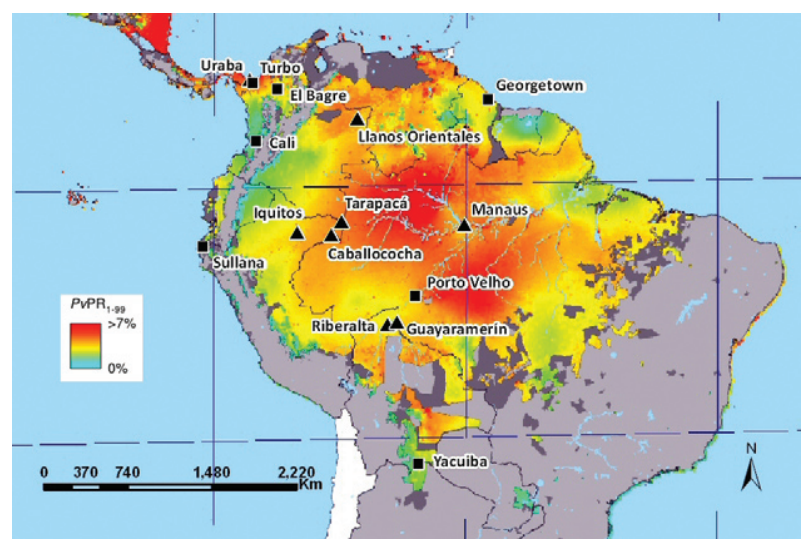

Map showing sites of recent in vivo studies of chloroquine treatment for Plasmodium vivax infections in South America (published between 2000-2013). Triangles indicate study sites where treatment failures have been reported, while squares indicate sites with no treatment failures observed over 28 days of follow-up. Note the close proximity between two sites in Colombia (Uraba and Turbo) and two sites in Bolivia (Riberalta and Guayaramerín). Clinical data are from Villalobos-Salcedo et al. (2000) (Porto Velho, Brazil), Soto et al. (2001) (Uraba and Llanos Orientales, Colombia), Baird et al. (2002) (Georgetown, Guyana), Ruebush et al. (2003) (Iquitos, Caballococha and Sullana, Peru), Alvarez et al. (2006) (Turbo and El Bagre, Colombia), de Santana Filho et el. (2007) (Manaus, Brazil), Osorio et al. (2007) (Tarapacá, Colombia), Carmona-Fonseca and Maestre (2009) (Turbo and El Bagre, Colombia), Añez et al. (2012) (Riberalta, Guayaramerín and Yacuiba, Bolivia) and Marques et al. (2013) (Manaus, Brazil). Shading patterns vary according to $P$. vivax malaria prevalence $(P v \mathrm{PR})$ data estimated by Gething et al. (2012).
A study comparing two CQ-PQ regimens in 73 Brazilian subjects showed no $P$. vivax recurrences up to 30 days after treatment (Villalobos-Salcedo et al. 2000). Nevertheless, most of these studies were underpowered to detect rare events due to small sample sizes. Furthermore, no CQ resistance data from in vivo studies are publicly available for $P$. vivax populations from other Latin American countries. In conclusion, the extent to which CQ-resistant $P$. vivax currently represents a significant threat to malaria control efforts in Latin America remains to be determined.

$C Q-P Q$ as a combination therapy - The reasons why $\mathrm{CQ}$ resistance tends to spread much slower in $P$. vivax than in Plasmodium falciparum remain open to speculation. The finding that PQ reverses CQ resistance in P. falciparum (Bray et al. 2005) suggests that a similar effect might occur in P. vivax isolates simultaneously exposed to both drugs [reviewed by Egan (2006)]. Over the past 60 years, CQ and PQ have been combined for the radical cure of $P$. vivax infections (i.e., eradication of blood stages and hepatic hypnozoites) in Latin America, but not in other regions where glucose-6-phosphate dehydrogenase (G6PD) deficiency is common due to the risk of severe PQ-induced haemolysis. CQ and PQ administration may be either simultaneous or sequential (PQ is usually given on day 28 of CQ treatment).

Therefore, CQ-resistant $P$. vivax first emerged and became widespread in Melanesia and parts of Southeast Asia, regions where PQ is not widely used because of the relatively high prevalence of G6PD deficiency in human populations (Howes et al. 2012). Moreover, a recent metaanalysis of four clinical trials carried out in Asia (Thailand and Pakistan) and Africa (Ethiopia), with a total of 1,423 patients, showed that CQ alone $(25 \mathrm{mg} / \mathrm{kg}$ over 3 days) is less effective against $P$. vivax asexual blood stages than CQ ( $25 \mathrm{mg} / \mathrm{kg}$ over 3 days) co-administered with PQ (15 mg of PQ base/day for 14 days), with a relative risk of parasite recrudescence of 1.92 (95\% confidence interval, 1.59-2.34) over 28 days of follow-up (Naing et al. 2010). Although these data suggest that $P$. vivax CQ resistance might not be a significant reason for concern in countries where CQ and PQ are routinely co-administered (Ferreira \& da Silva-Nunes 2010), there are clearly documented examples of $P$. vivax infections acquired in Guyana (Phillips et al. 1996) and Brazil (Marques et al. 2013) that were resistant to CQ-PQ combination therapy.

$P$. vivax $C Q$ resistance monitoring: a role for ex-vivo assays? - In addition to in vivo studies, $P$. vivax drug susceptibility may also be assessed ex vivo (testing parasites directly from infected patients) using short-term schizont maturation tests in the presence of increasing drug concentrations (Russell et al. 2003, Tasanor et al. 2006). Theoretically, in vitro analysis allows for a more objective assessment of drug resistance patterns than in vivo tests, with no influence of confounding factors such as patients' acquired immunity and antimalarial drug absorption and metabolism (Price et al. 2012). However, the comparison of data generated in different laboratories is affected by factors such as previous use of antimalarials, synchronicity of infection in the original clinical samples, 
time delays in processing these samples, variations in methods used for sample cryopreservation and thawing (if any) and differences in assay protocols, including methods for quantifying parasite growth (Russell et al. 2008). Moreover, no standard $P$. vivax strains with different drug resistance patterns are available for testing the reproducibility of assays in different laboratories. Interestingly, $P$. vivax displays striking stage-specific differences in CQ susceptibility: only the early ring stages are affected by CQ exposure, whereas late trophozoites and schizonts are almost completely CQ resistant (Russell et al. 2008).

Given these reasonable limitations, it is far from surprising that ex vivo drug susceptibility assays have rarely been used to evaluate CQ resistance in Latin American isolates of $P$. vivax. Nevertheless, two recent reports from Manaus revealed similar prevalence of CQ resistance in local $P$. vivax isolates. Both studies used similar assay protocols, with parasite growth being measured by antigen-capture dehydrogenase immunodetection assay and the same definition of CQ resistance [50\% inhibitory concentration $\left.\left(\mathrm{IC}_{50}\right)>100 \mathrm{nM}\right]$ was used. These studies showed a rate of $9.8 \%$ of CQ-resistant isolates among 132 clinical samples collected and processed between 2004-2007 (Pratt-Riccio et al. 2013) and 10.7\% among 112 samples collected between 2007-2008 (Chehuan et al. 2013). Such figures are consistent with the known recrudescence rate of $10.1 \%$ in the same area among $P$. vivax patients treated with CQ alone (de Santana Filho et al. 2007). Unfortunately, no further ex vivo CQ-resistance data are available for $P$. vivax samples from other regions of Brazil and elsewhere in Latin America.

Molecular mechanisms and markers of $P$. vivax $C Q$ resistance - Our knowledge about the mechanisms and molecular markers of CQ resistance in $P$. vivax remains limited and several factors may account for this. First, the absence of an effective and reproducible protocol for the continuous in vitro culture of this parasite, such as the one available for $P$. falciparum, has precluded the determination of clear-cut $\mathrm{IC}_{50}$ values for $\mathrm{CQ}$, which would allow establishing the associations between phenotypes and genotypes [for example, single-nucleotide variants (SNVs)]. Second, current in vivo studies for $P$. vivax are unable to distinguish a recrudescence of CQ-resistant parasites from a relapse or a new infection (which may be due to either a major or a minor parasite subpopulation in the original infection) (Price et al. 2012). As such, resulting associations between putative molecular markers of resistance and the pre-treatment of parasites may be spurious. Third, as efforts on genetic research on malaria have historically been spear-headed by studies on P. falciparum, we have largely used the resulting CQ resistance markers to infer those that would be appropriate for $P$. vivax. However, due to intrinsic biological dissimilarities, extrapolation from P. falciparum may not reflect the true drug resistance mechanisms of $P$. vivax, thus limiting the number of markers studied.

The main gene investigated within the context of CQ resistance in $P$. vivax is pvcrt, the homologue of the $P$. falciparum CQ resistance transporter ( $p f c r t$ ), in which a K76T substitution is known to be a crucial determinant of CQ resistance. A heterologous expression study in which pvcrt was transfected into P. falciparum and Dictyostelium discoideum showed that pvcrt overexpression was able to reduce CQ susceptibility in both organisms (Sá et al. 2006), suggesting that pvcrt may be able to modulate CQ transport and accumulation in P. vivax. In a different study, parasites from a traveller with severe vivax malaria acquired in the Brazilian Amazon showed a 22 -fold increase in the levels of pvcrt transcription compared to the parasites from three patients with uncomplicated malaria (Fernández-Becerra et al. 2009). Although some reports of drug resistance have originated from regions where severe vivax disease is common (Tjitra et al. 2008), a direct association between pvcrt overexpression, CQ resistance and malaria morbidity could not be established (Fernández-Becerra et al. 2009). Importantly, in vivo drug trials testing the clinical response of $P$. vivax to CQ failed to show a clear association between SNVs in pvcrt and treatment outcome (Nomura et al. 2001, Suwanarusk et al. 2007, OrjuelaSánchez et al. 2009, Ganguly et al. 2013), suggesting that SNVs in this gene are not appropriate molecular markers for the surveillance of CQ resistance in this parasite.

The $P$. vivax homologue of the P. falciparum multidrug resistance gene 1 (pvmdrl) has also been examined from the perspective of CQ resistance. In a study of natural parasite populations of $P$. vivax from Papua, Indonesia, where CQ resistance is highly prevalent, a sequence polymorphism in $p v m d r l$, translating into an Y976F substitution, was found in all patients presenting at a local health facility. The same substitution, however, was rare in Thailand, where $P$. vivax CQ resistance remains infrequent (Suwanarusk et al. 2007). In a different study, another sequence polymorphism in pvmdrl (F1076L) was identified in P. vivax samples from Thailand and Indonesia (Brega et al. 2005), countries where CQ resistance is known to occur, especially in the latter area. These polymorphisms are relatively infrequent in Latin America, where P. vivax CQ resistance remains relatively uncommon (Vargas-Rodríguez et al. 2012), though the Y976F substitution may reach a high prevalence in Eastern Amazonia (Gama et al. 2009). However, the absence of clinical correlates of CQ efficacy in these studies precludes the establishment of direct associations between the genetic polymorphisms analysed and treatment outcomes. The hypothesis that $p v m d r 1$ polymorphisms play a major role in modulating CQ responses is further challenged by the finding of CQ-resistant isolates with the wild-type version of the gene (Sá et al. 2005 ) or with other SNVs in the same gene (OrjuelaSánchez et al. 2009).

Genome-wide association studies can circumvent the limitations faced by single-gene approaches, as described previously, because they do not assume that the molecules are involved in resistance to any given drug. As such, these unconstrained methodologies may help deepen our knowledge about the mechanisms of CQ resistance and identify useful molecular markers for resistance surveillance. Such strategies are now beginning to be incorporated into $P$. vivax drug resistance research and they have already yielded interesting results. Recently, Chan et al. (2012) used whole-genome sequenc- 
ing (WGS) of five $P$. vivax isolates from Madagascar and Cambodia, obtained directly from patients' blood samples and from the monkey-adapted Belem strain. In doing so, these authors demonstrated that it is feasible to perform WGS of $P$. vivax field isolates and generated a catalogue of approximately 80,000 SNPs distributed throughout the genome that will enable large-scale genotyping studies and contribute to a better understanding of $P$. vivax traits, such as drug resistance (Chan et al. 2012). In a different work, a single uncultured isolate of $P$. vivax obtained from a patient from Peru with clinical malaria was subjected to WGS $(30 \times$ coverage) (Dharia et al. 2010). The authors reported that a number of genes contained high ratios of nonsynonymous-to-synonymous polymorphisms, including the $P$. vivax multidrug resistance-associated protein $(P v M R P 1)$, an $\mathrm{ABC}$ transporter previously shown to be linked to quinoline and antifolate responses in P. falciparum (Dharia et al. 2010). More recently, Bright et al. (2013) sequenced the entire genome of a $P$. vivax isolate derived from a traveller returning to Canada from Sudan and reported the identification of several SNVs as candidate molecular markers of drug resistance, which also included mutations in the pvmrpl gene (Bright et al. 2013).

A role for the Worldwide Antimalarial Resistance Network (WWARN) in Latin America - The WWARN (wwarn.org) is a global network of academic experts aiming to improve the extent and quality of data, fill regional research and data gaps and strengthen resources to collate available data, with the ultimate goal of producing reliable information to help identify and track the global spread of malaria drug resistance (Sibley et al. 2008). Established in 2009 with funds from the Bill and Melinda Gates Foundation, WWARN has its secretariat in Oxford (United Kingdom), with regional centres in Bangkok (Thailand), Dakar (Senegal), Nairobi (Kenya), Cape Town (South Africa) and, since 2012, a Latin American representation based in São Paulo, Brazil.

As a multidisciplinary network, WWARN focuses on five aspects of drug resistance: clinical, molecular, in vitro, pharmacology and antimalarial quality. Over the past five years, WWARN has developed customised tools and services designed to facilitate quality-assured data collection, analysis and reporting. Over 350 clinical, molecular, in vitro and pharmacokinetic data sets (comprising 90,000 patient records, mainly from falciparum malaria studies) have been uploaded into the WWARN repository. This repository allows for automated quality assurance and the integration of data from many different sources, building a comprehensive picture of resistance while protecting the rights of the data owners.

In addition to the data repository, WWARN has developed several freely available tools, services and training materials to support the malaria research community (wwarn.org/toolkit). One of these online tools is the Parasite Clearance Estimator (PCE) software (Flegg et al. 2011) (available from wwarn.org/pce/), which provides standardised estimates of parasite clearance rates in terms of slope half-life (Flegg et al. 2013). PCE has been used to compare data derived from several clinical trials of artemisinin combination therapies (ACTs) for falciparum malaria and can also be used to analyse $P$. vivax clearance rates following CQ treatment.

Another popular WWARN software is the In Vitro Analysis and Reporting Tool (IVART) (Woodrow et al. 2013) (available from wwarn.org/toolkit/data-management/ivart). IVART automatically generates $\mathrm{IC}_{50}$ estimates and plots data obtained with different in vitro assay formats and readouts. Although IVART has been extensively used in $P$. falciparum drug resistance studies, it can also be applied to ex vivo schizont maturation inhibition assays of $P$. vivax.

Mapping and containing $P$. vivax CQ resistance in Latin America poses a major challenge for local researchers and regional networks. Presently, drug resistance surveillance activities have mainly focused on ACT efficacy against $P$. falciparum, with scarce data on vivax malaria therapies (de Santana Filho et al. 2007). Accordingly, the newly established WWARN representation in Latin America has been collaborating with researchers to collate and analyse available in vivo and in vitro data (both published and unpublished) and develop an accurate picture of $P$. vivax CQ resistance in the region. Moreover, WWARN is working in close collaboration with local clinical and laboratory-oriented researchers to facilitate the integration and harmonisation of study protocols, aiming to generate high-quality in vivo data and clinical samples for the ex vivo and molecular characterisation of CQ-resistant $P$. vivax isolates using state-of-the-art techniques. This multidisciplinary effort was launched during a WWARN-sponsored regional workshop held in São Paulo in August 2013 and now includes malaria experts from five Latin American countries.

WWARN invites Latin American researchers to become involved in this initiative, both by sharing data and study protocols and by offering suggestions and comments to improve the design of new studies of malaria drug resistance in the region. Working together, we can make a difference.

Concluding remarks - Recent advances in our capacity to generate genome data de novo and to rapidly analyse these data have opened up unprecedented opportunities for deepening our knowledge about drug resistance in $P$. vivax. It has already been possible to identify genetic signatures of positive selection in areas of CQ resistance using genome-wide association studies. In the future, it should also be possible to compare the genomes of confirmed CQ-resistant parasites with those from sensitive ones or to identify CQ resistance loci using Linkage Group Selection on genetic crosses. Ultimately, such strategies will allow the identification of new putative molecular markers of CQ resistance in $P$. vivax, the value of which will rest on the demonstration that such markers effectively predict the outcome of treatment in vivo.

\section{ACKNOWLEDGEMENTS}

To Dr Márcia C de Castro (Harvard School of Public Health), for help in preparing the Figure, and to Prof Carol $\mathrm{H}$ Sibley and Dr Philippe Guérin, for their comments on earlier drafts of this article. 


\section{REFERENCES}

Alvarez G, Piñeros JG, Tobón A, Ríos A, Maestre A, Blair S, Carmona-Fonseca J 2006. Efficacy of three chloroquine-primaquine regimens for treatment of Plasmodium vivax malaria in Colombia. Am J Trop Med Hyg 75: 605-609.

Añez A, Navarro-Costa D, Yucra O, Garnica C, Melgar V, Moscoso M, Arteaga R, Nakao G 2012. Therapeutic response of Plasmodium vivax to chloroquine in Bolivia. Biomedica 32: 527-535.

Arevalo-Herrera M, Quiñones ML, Guerra C, Céspedes N, Giron S, Ahumada M, Piñeros JG, Padilla N, Terrientes Z, Rosas A, Padilla JC, Escalante AA, Beier JC, Herrera S 2012. Malaria in selected non-Amazonian countries of Latin America. Acta Trop 121: $303-314$.

Arias AE, Corredor A 1989. Low response of Colombian strains of Plasmodium vivax to classical antimalarial therapy. Trop Med Parasitol 40: 21-23.

Baird JK 2009. Resistance to therapies for infection by Plasmodium vivax. Clin Microbiol Rev 22: 508-534.

Baird JK, Leksana B, Masbar S, Fryauff DJ, Sutanihardja MA, Suradi, Wignall FS, Hoffman SL 1997. Diagnosis of resistance to chloroquine by Plasmodium vivax: timing of recurrence and whole blood chloroquine levels. Am J Trop Med Hyg 56: 621-626.

Baird JK, Maguire JD, Price RN 2012. Diagnosis and treatment of Plasmodium vivax malaria. Adv Parasitol 80: 203-270.

Baird JK, Tiwari T, Martin GJ, Tamminga CL, Prout TM, Tjaden J, Bravet PP, Rawlins S, Ferrel M, Carucci D, Hoffman SL 2002. Chloroquine for the treatment of uncomplicated malaria in Guyana. Ann Trop Med Parasitol 96: 339-348.

Battle KE, Gething PW, Elyazar IR, Moyes CL, Sinka ME, Howes RE, Guerra CA, Price RN, Baird KJ, Hay SI 2012. The global public health significance of Plasmodium vivax. Adv Parasitol 80: 1-111.

Bray PG, Deed S, Fox E, Kalkanidis M, Mungthin M, Deady LW, Tilley L 2005. Primaquine synergises the activity of chloroquine against chloroquine-resistant $P$. falciparum. Biochem Pharmacol 70: 1158-1166.

Brega S, Meslin B, de Monbrison F, Severini C, Gradoni L, Udomsangpetch R, Sutanto I, Peyron F, Picot S 2005. Identification of the Plasmodium vivax mdr-like gene ( $p v m d r l)$ and analysis of single-nucleotide polymorphisms among isolates from different areas of endemicity. J Infect Dis 191: 272-277.

Bright AT, Alenazi T, Shokoples S, Tarning J, Paganotti GM, White NJ, Houston S, Winzeler EA, Yanow SK 2013. Genetic analysis of primaquine tolerance in a patient with relapsing vivax malaria. Emerg Infect Dis 19: 802-805.

Carmona-Fonseca J, Maestre A 2009. Prevention of Plasmodium vivax malaria recurrence: efficacy of the standard total dose of primaquine administered over 3 days. Acta Trop 112: 188-192.

Castillo CM, Osorio LE, Palma GI 2002. Assessment of therapeutic response of Plasmodium vivax and Plasmodium falciparum to chloroquine in a malaria transmission free area in Colombia. Mem Inst Oswaldo Cruz 97: 559-562.

Chan ER, Menard D, David PH, Ratsimbasoa A, Kim S, Chim P, Do C, Witkowski B, Mercereau-Puijalon O, Zimmerman PA, Serre D 2012. Whole genome sequencing of field isolates provides robust characterization of genetic diversity in Plasmodium vivax. PLoS Negl Trop Dis 6: e1811.

Chehuan YF, Costa MR, Costa JS, Alecrim MG, Nogueira F, Silveira H, Brasil LW, Melo GC, Monteiro WM, Lacerda MV 2013. In vitro chloroquine resistance for Plasmodium vivax isolates from the western Brazilian Amazon. Malar J 12: 226. da Silva-Nunes M, Moreno M, Conn JE, Gamboa D, Abeles S, Vinetz JM, Ferreira MU 2012. Amazonian malaria: asymptomatic human reservoirs, diagnostic challenges, environmentally driven changes in mosquito vector populations and the mandate for sustainable control strategies. Acta Trop 121: 281-291.

de Santana Filho FS, Arcanjo AR, Chehuan YM, Costa MR, Martinez-Espinosa FE, Vieira JL, Barbosa MG, Alecrim WD, Alecrim MG 2007. Chloroquine-resistant Plasmodium vivax, Brazilian Amazon. Emerg Infect Dis 13: 1125-1126.

Dharia NV, Bright AT, Westenberger SJ, Barnes SW, Batalov S, Kuhen K, Borboa R, Federe GC, McClean CM, Vinetz JM, Neyra V, Llanos-Cuentas A, Barnwell JW, Walker JR, Winzeler EA 2010. Whole-genome sequencing and microarray analysis of ex vivo Plasmodium vivax reveal selective pressure on putative drug resistance genes. Proc Natl Acad Sci USA 107: 20045-20050.

Egan TJ 2006. Chloroquine and primaquine: combining old drugs as a new weapon against falciparum malaria? Trends Parasitol 22: 235-237.

Fernández-Becerra C, Pinazo MJ, González A, Alonso PL, del Porti1lo HA, Gascón J 2009. Increased expression levels of the pvcrt-o and pvmdrl genes in a patient with severe Plasmodium vivax malaria. Malar J 8: 55.

Ferreira MU, da Silva-Nunes M 2010. Evidence-based public health and prospects for malaria control in Brazil. J Infect Dev Ctries 4: $533-545$.

Flegg JA, Guerin PJ, Nosten F, Ashley EA, Phyo AP, Dondorp AM, Fairhurst RM, Socheat D, Borrmann S, Bjorkman A, Martensson A, Mayxay M, Newton PN, Bethell D, Se Y, Noedl H, Diakite M, Djimde AA, Hien TT, White NJ, Stepniewska K 2013. Optimal sampling designs for estimation of Plasmodium falciparum clearance rates in patients treated with artemisinin derivatives. Malar J 12: 411

Flegg JA, Guerin PJ, White NJ, Stepniewska K 2011. Standardizing the measurement of parasite clearance in falciparum malaria: the parasite clearance estimator. Malar J 10: 339.

Gama BE, de Oliveira NKA, de Souza JM, Daniel-Ribeiro CT, Ferreira-da-Cruz MF 2009. Characterisation of pvmdrl and pvdhfr genes associated with chemoresistance in Brazilian Plasmodium vivax isolates. Mem Inst Oswaldo Cruz 104: 1009-1011.

Ganguly S, Saha P, Guha SK, Das S, Bera DK, Biswas A, Kundu PK, Saha B, Ray K, Maji AK 2013. In vivo therapeutic efficacy of chloroquine alone or in combination with primaquine against vivax malaria in Kolkata, West Bengal, India and polymorphism in pvmdrl and pvcrt-o genes. Antimicrob Agents Chemother 57: 1246-1251.

Garavelli PL, Corti E 1992. Chloroquine resistance in Plasmodium vivax: the first case in Brazil. Trans R Soc Trop Med Hyg 86: 128.

Gething PW, Elyazar IR, Moyes CL, Smith DL, Battle KE, Guerra CA, Patil AP, Tatem AJ, Howes RE, Myers MF, George DB, Horby P, Wertheim HF, Price RN, Müeller I, Baird JK, Hay SI 2012. A long neglected world malaria map: Plasmodium vivax endemicity in 2010. PLoS Negl Trop Dis 6: e1814.

Howes RE, Piel FB, Patil AP, Nyangiri OA, Gething PW, Dewi M, Hogg MM, Battle KE, Padilla CD, Baird JK, Hay SI 2012. G6PD deficiency prevalence and estimates of affected populations in malaria endemic countries: a geostatistical model-based map. PLoS Med 9: e1001339.

Marques MM, Costa MR, Santana Filho FS, Vieira JL, Nascimento MT, Brasil LW, Nogueira F, Silveira H, Reyes-Lecca RC, Monteiro WM, Lacerda MV, Alecrim MG 2013. Plasmodium vivax chloroquine resistance and anemia in the western Brazilian Amazon. Antimicrob Agents Chemother 58: 342-347. 
Naing C, Aung K, Win DK, Wah MJ 2010. Efficacy and safety of chloroquine for treatment in patients with uncomplicated Plasmodium vivax infections in endemic countries. Trans $R$ Soc Trop Med Hyg 104: 695-705.

Nomura T, Carlton JM, Baird JK, del Portillo HA, Fryauff DJ, Rathore D, Fidock DA, Su X, Collins WE, McCutchan TF, Wootton JC, Wellems TE 2001. Evidence for different mechanisms of chloroquine resistance in 2 Plasmodium species that cause human malaria. J Infect Dis 183: 1653-1661.

Orjuela-Sánchez P, de Santana Filho FS, Machado-Lima A, Chehuan YF, Costa MR, Alecrim MG, del Portillo HA 2009. Analysis of single-nucleotide polymorphisms in the crt-o and $m d r l$ genes of Plasmodium vivax among chloroquine-resistant isolates from the Brazilian Amazon Region. Antimicrob Agents Chemother 53: 3561-3564.

Osorio L, Pérez LP, González IJ 2007. Assessment of the efficacy of antimalarial drugs in Tarapacá, in the Colombian Amazon Basin. Biomedica 27: 133-140.

Phillips EJ, Keystone JS, Kain KC 1996. Failure of combined chloroquine and high-dose primaquine therapy for Plasmodium vivax malaria acquired in Guyana, South America. Clin Infect Dis 23: 1171-1173.

Pratt-Riccio LR, Chehuan YF, Siqueira MJ, Alecrim MG, BiancoJunior C, Druilhe P, Brasseur P, Ferreira-da-Cruz MF, Carvalho LJ, Daniel-Ribeiro CT 2013. Use of a colorimetric (DELI) test for the evaluation of chemoresistance of Plasmodium falciparum and Plasmodium vivax to commonly used anti-plasmodial drugs in the Brazilian Amazon. Malar J 12: 281.

Price RN, Auburn S, Marfurt J, Cheng Q 2012. Phenotypic and genotypic characterisation of drug-resistant Plasmodium vivax. Trends Parasitol 28: 522-529.

Price RN, Tjitra E, Guerra CA, Yeung S, White NJ, Anstey NM 2007. Vivax malaria: neglected and not benign. Am J Trop Med Hyg 77 (Suppl. 6): 79-87.

Rieckmann KH, Davis DR, Hutton DC 1989. Plasmodium vivax resistance to chloroquine? Lancet 2: 1183-1184.

Ruebush TK 2nd, Zegarra J, Cairo J, Andersen EM, Green M, Pillai DR, Marquiño W, Huilca M, Arévalo E, Garcia C, Solary L, Kain KC 2003. Chloroquine-resistant Plasmodium vivax malaria in Peru. Am J Trop Med Hyg 69: 548-552.

Russell B, Chalfein F, Prasetyorini B, Kenangalem E, Piera K, Suwanarusk R, Brockman A, Prayoga P, Sugiarto P, Cheng Q, Tjitra E, Anstey NM, Price RN 2008. Determinants of in vitro drug susceptibility testing of Plasmodium vivax. Antimicrob Agents Chemother 52: 1040-1045.

Russell BM, Udomsangpetch R, Rieckmann KH, Kotecka BM, Coleman RE, Sattabongkot J 2003. Simple in vitro assay for determining the sensitivity of Plasmodium vivax isolates from fresh human blood to antimalarials in areas where $P$. vivax is endemic. Antimicrob Agents Chemother 47: 170-173.

Sá JM, Nomura T, Neves JD, Baird JK, Wellems TE, del Portillo HA 2005. Plasmodium vivax: allele variants of the $m d r l$ gene do not associate with chloroquine resistance among isolates from Brazil, Papua and monkey-adapted strains. Exp Parasitol 109: 256-259.

Sá JM, Yamamoto MM, Fernandez-Becerra C, de Azevedo MF, Papakrivos J, Naudé B, Wellems TE, del Portillo HA 2006. Expression and function of pvcrt-o, a Plasmodium vivax ortholog of pfcrt, in Plasmodium falciparum and Dictyostelium discoideum. Mol Biochem Parasitol 150: 219-228.

Sibley CH, Barnes KI, Watkins WM, Plowe CV 2008. A network to monitor antimalarial drug resistance: a plan for moving forward. Trends Parasitol 24: 43-48.

Soto J, Toledo J, Gutierrez P, Luzz M, Llinas N, Cedeño N, Dunne M, Berman J 2001. Plasmodium vivax clinically resistant to chloroquine in Colombia. Am J Trop Med Hyg 65: 90-93.

Suwanarusk R, Russell B, Chavchich M, Chalfein F, Kenangalem E, Kosaisavee V, Prasetyorini B, Piera KA, Barends M, Brockman A, Lek-Uthai U, Anstey NM, Tjitra E, Nosten F, Cheng Q, Price RN 2007. Chloroquine resistant Plasmodium vivax: in vitro characterisation and association with molecular polymorphisms. PLOS ONE 2: e1089.

Tasanor O, Ruengweerayut R, Sirichaisinthop J, Congpuong K, Wernsdorfer WH, Na-Bangchang K 2006. Clinical-parasitological response and in vitro sensitivity of Plasmodium vivax to chloroquine and quinine on the western border of Thailand. Trans $R$ Soc Trop Med Hyg 100: 410-418.

Tjitra E, Anstey NM, Sugiarto P, Warikar N, Kenangalem E, Karyana M, Lampah DA, Price RN 2008. Multidrug-resistant Plasmodium vivax associated with severe and fatal malaria: a prospective study in Papua, Indonesia. PLoS Med 5: e128.

Vargas-Rodríguez RC, Bastos MS, Menezes MJ, Orjuela-Sánchez P, Ferreira MU 2012. Single-nucleotide polymorphism and copy number variation of the multidrug resistance-1 locus of Plasmodium vivax: local and global patterns. Am J Trop Med Hyg 87: 813-821.

Villalobos-Salcedo JM, Tada MS, Kimura E, Menezes MJ, da Silva LHP 2000. In vivo sensitivity of Plasmodium vivax isolates from Rondônia (western Amazon Region, Brazil) to regimens including chloroquine and primaquine. Ann Trop Med Parasitol 94: 749-758.

Woodrow CJ, Dahlstrom S, Cooksey R, Flegg JA, Le Nagard H, Mentre F, Murillo C, Menard D, Nosten F, Sriprawat K, Musset L, Quashie NB, Lim P, Fairhurst RM, Nsobya SL, Sinou V, Noedl H, Pradines B, Johnson JD, Guerin PJ, Sibley CH, Le Bras J 2013. High-throughput analysis of antimalarial susceptibility data by the World Wide Antimalarial Resistance Network (WWARN) in vitro analysis and reporting tool. Antimicrob Agents Chemother 57: 3121-3130. 